\title{
CO-CRYSTALS OF CARVEDILOL: PREPARATION, CHARACTERIZATION AND EVALUATION
}

\author{
RAJU THENGE ${ }^{*}$, RAKESH PATEL ${ }^{1}$, NANDU KAYANDE ${ }^{2}$, NILESH MAHAJAN ${ }^{3}$
}

${ }^{1}$ School of Pharmacy, Dr. A. P. J. Abdul Kalam University, Indore (M. P), India, ${ }^{2}$ Thakur Shivkumar Singh Memorial Pharmacy College, Burhanpur, (M. P), India, ${ }^{3}$ Dadasaheb Balpande College of Pharmacy, Besa-Nagpur, Maharashtra, India

Email: rajuthenge11@gmail.com

Received: 09 Sep 2019, Revised and Accepted: 01 Nov 2019

ABSTRACT

Objective: Carvedilol an antihypertensive drug, exhibits poor solubility and dissolution rate. Hence an attempt has been made to prepare the Cocrystals of Carvedilol to increase the solubility and dissolution rate.

Methods: The Co-crystals of Carvedilol were prepared using coformer such as succinic acid, fumaric acid and oxalic acid by Solvent evaporation method. The prepared Co-crystals were evaluated for solubility, dissolution rate and micrometric properties. The Co-crystals were characterized by scanning electronic Microscopy (SEM), FT-Infrared Spectroscopy (FTIR), Differential Scanning Calorimetry (DSC) and X-ray Diffractometry (XRD).

Results: SEM of pure carvedilol and Cocrystals morphology clearly showed the formation of a new solid phase with the coformer. The FT-IR spectra indicate the shifting of characteristic peak in the Co-crystals but does not show any interaction between the co-former used. DSC data showed the change in the endotherm with the melting point of Co-crystals. XRD spectra indicate the notified difference in the $2 \theta$ and the intensity of the peaks. Solubility of CAR-SA Cocrystals (2.225 \pm 0.35$)$, CAR-FA Cocrystals $(1.880 \pm 0.20)$ and CAR-OA Cocrystals $(1.128 \pm 0.23)$ was markedly improved compared to pure Carvedilol $(0.376 \pm 0.06)$. Thus the increased in dissolution rate for CAR-SA Cocrystals (93.72 \%). was highest whereas CAR-FA Cocrystals (91.56 \%), CAR-OA Cocrystals (88.93\%) compared to pure Carvedilol (40.3) within 60 Min. The Carvedilol cocrystals were also showed improvement in the flow properties compare to pure Carvedilol.

Conclusion: Hence the Co-crystal formation could be helpful to improve the solubility, dissolution and micromeritic properties of Carvedilol.

Keywords: Carvedilol, Co-crystals, Solubility, FT-IR, Dissolution rate, Micromeritic properties

(C) 2020 The Authors. Published by Innovare Academic Sciences Pvt Ltd. This is an open access article under the CC BY license (http://creativecommons.org/licenses/by/4.0/) DOI: http://dx.doi.org/10.22159/ijap.2020v12i1.35640. Journal homepage: https://innovareacademics.in/journals/index.php/ijap

\section{INTRODUCTION}

For hydrophobic drugs, the dissolution process act as the ratecontrolling step and which determines the rate and degree of absorption. Consequently, many hydrophobic drugs show erratic and incomplete absorption from the gastrointestinal tract of animals and humans. Thus, one of the major challenges of drug development today is poor solubility, as an estimated $40 \%$ of all newly developed drugs are poorly soluble or insoluble in water [1]. In addition, up to $50 \%$ of orally administered drug compounds suffer from formulation problems related to their low solubility and high lipophilicity [2-4]. Bioavailability of poorly water-soluble hydrophobic drugs [Class II in Biopharmaceutics Classification System] is limited by their solubility and dissolution rate. As a result, more than $40 \%$ of new candidates entering the drug development pipeline fail because of non-optimal biopharmaceutical properties [5]. Many approaches have been adopted for improving the aqueous solubility of drugs including micronization, emulsifications, solubilization using co-solvents, and the of polymer drug vehicles for delivery of poorly water soluble drug. Although these techniques have been shown to be effective at enhancing oral bioavailability, the success of these approaches dependent on the specific physiochemical nature of the molecule being studied [6]. Over the last decade, there has been growing interest in the design of pharmaceutical co-crystals, which has emerged as a potential method for enhancing the bioavailability of drugs with low solubility. Co-crystal can be defined in a number of ways. A restrictive definition utilized by that co-crystals are structurally homogenous crystalline materials containing two or more components present in definite stoichometric amounts. The cocrystals components are discrete neutral molecular reactants which are solid at ambient temperature. Based on this definition of cocrystal, a pharmaceutical co-crystal means co-crystals with one of the co-crystal component as an Active Pharmaceutical Ingredient (API) and other components are called conformers [7, 8]. Co-crystals incorporate pharmaceutically acceptable guest molecules into the crystal lattice along with active pharmaceutical ingredient (API). Cocrystal has gained attention as attractive alternate solid forms for drug development. Physicochemical properties of pharmaceutical can be improved by obtaining co-crystals using co-crystallization. Co-crystallization with pharmaceutically acceptable compounds does not affect pharmacological activity of the API but can improve physical properties, such as solubility, hygroscopisity, compaction behavior [9].

The present study deals with preparation and characterization of Carvedilol cocrystals using suitable coformer to improve the solubility, dissolution rate and micromeritic properties.

\section{MATERIALS AND METHODS}

Carvedilol obtained as a gift sample from Glenmark generics, limited, Goa, India. Succinic acid, fumaric acid, oxalic acid and solvents were purchased from SD Fine Chemical Mumbai. All the solvents used are of analytical grade.

\section{Preparation of co-crystals of carvedilol}

Carvedilol Co-crystals were prepared by using the solvent evaporation method. Carvedilol and coformer (1:1 molar ratio) were dissolved in methanol and left aside for slow evaporation. The fine crystals were obtained after $24 \mathrm{~h}$. The prepared co-crystals were collected, dried and store in desiccators at room temp for the further studies [10].

\section{Characterization of co-crystals}

\section{Scanning electron microscopy (SEM)}

Photomicrographs of pure Carvedilol and its Cocrystal were obtained from scanning electron microscope (JEOL 5400, Japan.). The surface morphology of the sample was accessed by SEM. The samples were sputter coated with gold before scanning, operated at an acceleration voltage of $15 \mathrm{kV}[11,12]$.

\section{Fourier transform infrared spectroscopy (FTIR)}

The FTIR spectra of pure Carvedilol and its Cocrystal were obtained using Fourier Transform Infrared Spectrometer (Thermo-Fischer, Switzerland). The sample was placed in the holder and spectra were 
recorded in the range of $4000-400 \mathrm{~cm}^{-1}$. The FT-IR studies are generally carried out to check the interaction between drug and coformer [11, 12].

\section{Differential scaninng calorimetry (DSC)}

Thermograms of the pure Carvedilol and its Cocrystal were obtained from (Metlar Toledo, DSC 1) Phase transition of the Carvedilol and its Co-crystals were analyzed by DSC. Samples were sealed in an aluminum crucible and heated at the rate of $10{ }^{\circ} \mathrm{C} / \mathrm{min}$ up to $300{ }^{\circ} \mathrm{C}$ under a nitrogen atmosphere $(40 \mathrm{ml} / \mathrm{min})$. The exact peak temperature and melting point and heat of fusion were automatically calculated [11, 12].

\section{Powder X-ray diffraction studies}

Powder X-ray diffraction patterns (XRD) of the pure Carvedilol and its Cocrystals were monitored with an x-ray diffractometer (Bruker, D8 advance, Germany.) using copper as an x-ray target, a voltage of $40 \mathrm{KV}$, a current of $25 \mathrm{~mA}$ and with $2.28970 \AA$ \& wavelengths. The samples were analyzed over the $2 \theta$ range of $10.01-99.99^{\circ}$ with a scanning step size of $0.020(2 \theta)$ and scan step time of 0.8 second $[11,12]$.

\section{Solubility study of carvedilol}

An excess quantity of pure Carvedilol and Cocrystals were dissolved in $10 \mathrm{ml}$ vial containing distilled water. The samples were stirred for $24 \mathrm{~h}$ at $37 \pm 0.5^{\circ} \mathrm{C}$ with the help of magnetic stirrer with hot plate at $70 \mathrm{rpm}$. The sample was then filtered through a whatman filter paper (No. 42) and the amount of drug dissolved was analyzed spectrophotometrically at $285 \mathrm{~nm}$ [13].

\section{Micromeretic study}

The flow properties of pure Carvedilol and its Co-crystals were determined in terms of angle of repose, bulk density, tapped density, Carr's Index and Hausner's ratio. Angle of repose was determined by the fixed funnel method whereas Carr's Index and Hausner's ratio were calculated from the bulk and tapped densities. Hausner's ratio was taken as a ratio of tapped density to bulk density. Carr's Index was calculated according to the given equation [14].

\section{Angle of repose}

Angle of repose has been used to characterize the flow properties of solids. It is a characteristic related to inter particulate friction or resistance to movement between particles. This is the maximum angle possible between the surface of pile of powder or granules and the horizontal plane [14].

$$
\varnothing=\tan ^{-1} h / r
$$

Where, $\varnothing=$ angle of repose

$h=$ height of heap, $r$ = radius of base of heap circle.

\section{Compressibility index and hausner's ratio}

In recent years, compressibility index and the closely related Hausner's ratio have become the simple, fast and popular methods of predicting powder flow characteristics. Compressibility index has been proposed as an indirect measure of bulk density, size and shape, surface area, moisture content, and cohesiveness of materials because all of these can influence the observed compressibility index. The compressibility index and Hausner's ratio are determined by measuring both bulk density and the tapped density of a powder [14].

$$
\text { Carrs Index }=\mathrm{W} 1-\mathrm{W} 2 / \mathrm{W} 1 * 10
$$

Where, $\mathrm{W} 1=$ Tapped density, $\mathrm{W} 2=$ Bulk density

$$
\text { Hausners ratio }=\text { Tapped density } / \text { bulk density. }
$$

\section{Dissolution studies}

Dissolution studies of pure Carvedilol and Co-crystals were carried out using USP Dissolution Testing Apparatus Type II (Electrolab dissolution tester TDT-081, India.) Pure Carvedilol and Co-crystals filled into a muslin cloth and tied it to the paddle with thread. The phosphate buffer $\mathrm{pH}$ 6.8. (900 ml) was used as a dissolution medium. The paddles were stirred at $50 \mathrm{rpm}$, and the temperature was maintained at $37 \pm 0.5{ }^{\circ} \mathrm{C}$ after a specific time interval, $5 \mathrm{ml}$ of aliquot was withdrawn and replaced with the same amount of dissolution medium. The samples were suitably diluted with dissolution medium and absorbance was measured using UV Spectrophotometer (UV-1800, Shimadzu, Japan.) at $285 \mathrm{~nm}$ [15].

\section{RESULTS AND DISCUSSION}

\section{Characterization of Co-crystals}

\section{Scanning electron microscopy (SEM)}

The morphology of pure Carvedilol and Co-crystals with different conformers has studied using scanning electron microscopy. The pure carvedilol have showed the plate type crystals whereas the Cocrystals prepared using coformer showed the irregular shape of crystals. This change in the crystal habits indicates the formation of Co-crystals.

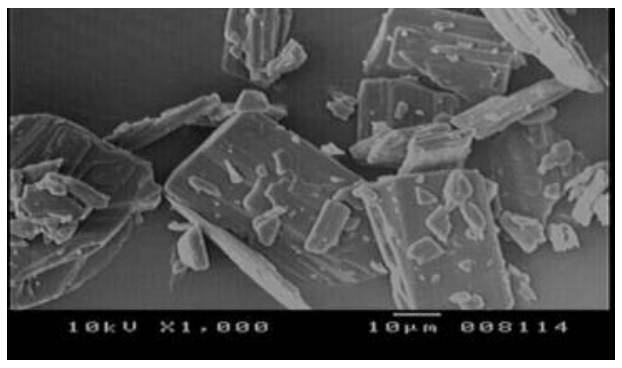

Fig. 1(A): SEM of pure carvedilol

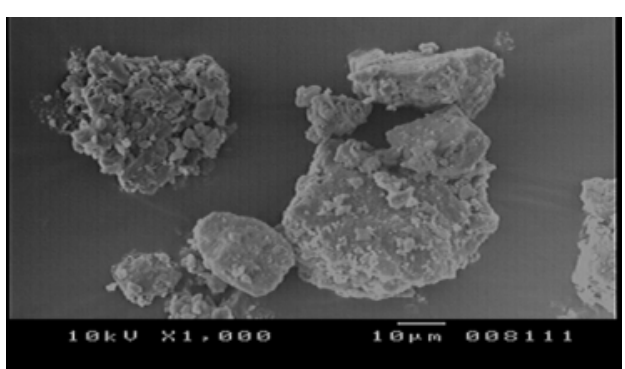

Fig. 1(B): CAR-SA Co-crystals

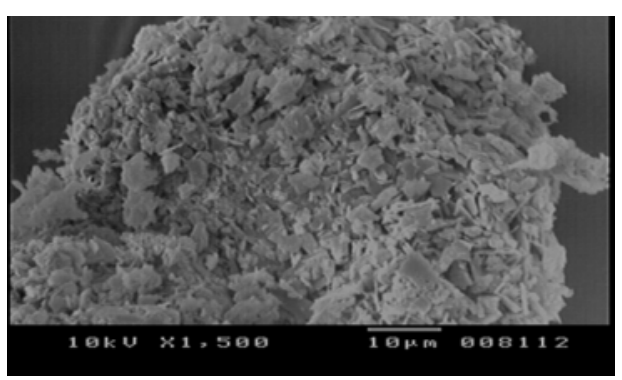

Fig. 1(C): CAR-FA Co-crystals

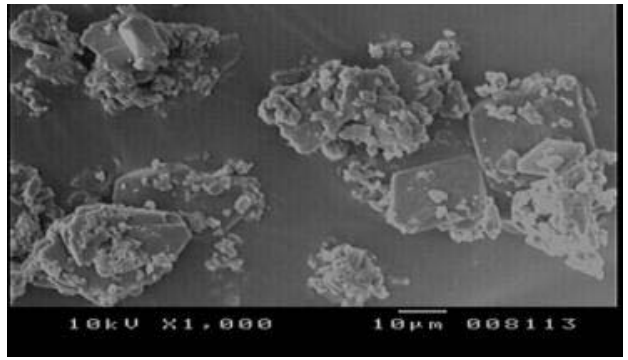

Fig. 1(D): CAR-OA Co-crystals 


\section{Fourier transform infrared spectroscopy (FTIR)}

FTIR spectra is useful to check the interaction between the drug and coformer. The characteristic peaks of Carvedilol appeared at $2923.4 \mathrm{~cm}$ ${ }^{1}$ (C-H stretching), $1594.1 \mathrm{~cm}-1$ (C=C stretching), $3060.2 \mathrm{~cm}-1$ (-C-Hstertching), $3344.9^{\mathrm{cm}-1}$ (O-H stretching), $752.0^{\mathrm{cm}-1}$ (=C-H stretching), $1100.1^{\mathrm{cm}-1}$ (C-O-C stretching) and $1217.6^{\mathrm{cm}-1}$ (C-N stretching). The FTIR spectra cocrystals with succinic acid show the ( $\mathrm{C}=\mathrm{C}$ stretching) at 3022.1 ${ }^{\mathrm{cm}-1}$ and (C-H-stretching) at $3402.8^{\mathrm{cm}-1}$. For Cocrystal with fumaric acid show (C-H-stretching) at $3047.9 \mathrm{~cm}-1$ and rest of peaks as that of pure carvedilol. The spectra of cocrystal with oxalic acid show (C-Hstretching) at $3081.3^{\mathrm{cm}-1}$ and (O-H-stretching) at $3400.09^{\mathrm{cm}-1}$. There is no appearance of new peaks in the spectra of cocrystals indicate that no interaction between the drug and polymer. The FTIR spectra of prepared co-crystals indicate shifting in the functional group compared to pure Carvedilol. This shift in the Co-crystals indicates the formation of new bonding during the formation of Co-crystals [16]. Thus FTIR spectroscopy helpful for the confirmation of the formation of co-crystals.

Table 1: FTIR data of pure carvedilol and its cocrystals

\begin{tabular}{|c|c|c|c|c|}
\hline Characteristics peaks & Pure carvedilol & $\begin{array}{l}\text { CAR-SA } \\
\text { Co-crystal }\end{array}$ & $\begin{array}{l}\text { CAR-FA } \\
\text { Co-crystal }\end{array}$ & $\begin{array}{l}\text { CAR-OA } \\
\text { Co-crystal }\end{array}$ \\
\hline C-H Stretching & 2923.4 & 2927.2 & 2927.9 & 2932.5 \\
\hline $\mathrm{C}=\mathrm{C}$ Stretching & 1594.1 & 1594.6 & 1597.4 & 1594.8 \\
\hline -C-H-Stretching & 3060.2 & 3022.8 & 3047.9 & 3081.3 \\
\hline O-H Stretching & 3344.9 & 3402.1 & 3345.6 & 3400.9 \\
\hline$=\mathrm{C}-\mathrm{H}$ Stretching & 752.0 & 751.1 & 750.9 & 751.9 \\
\hline C-O-C Stretching & 1100.1 & 1099.9 & 1099.8 & 1100.3 \\
\hline C-N Stretching & 1217.6 & 1220.4 & 1219.5 & 1220.1 \\
\hline
\end{tabular}

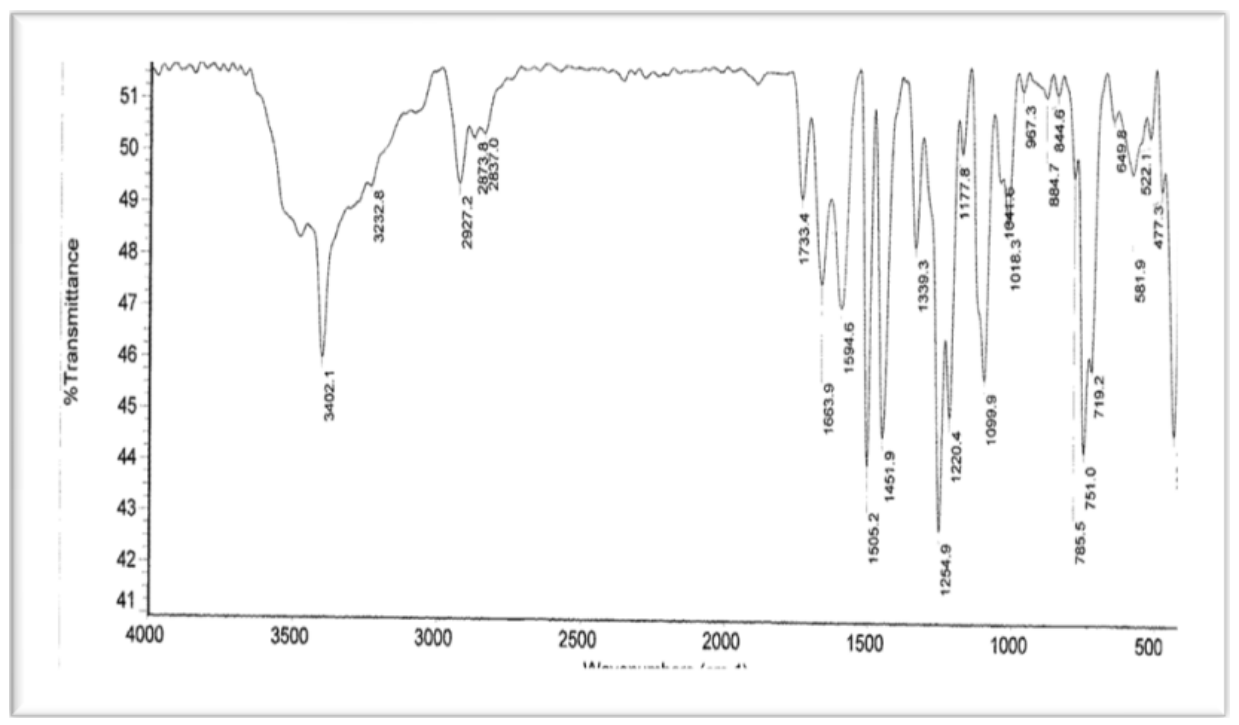

Fig. 2(A): FTIR spectra of pure carvedilol

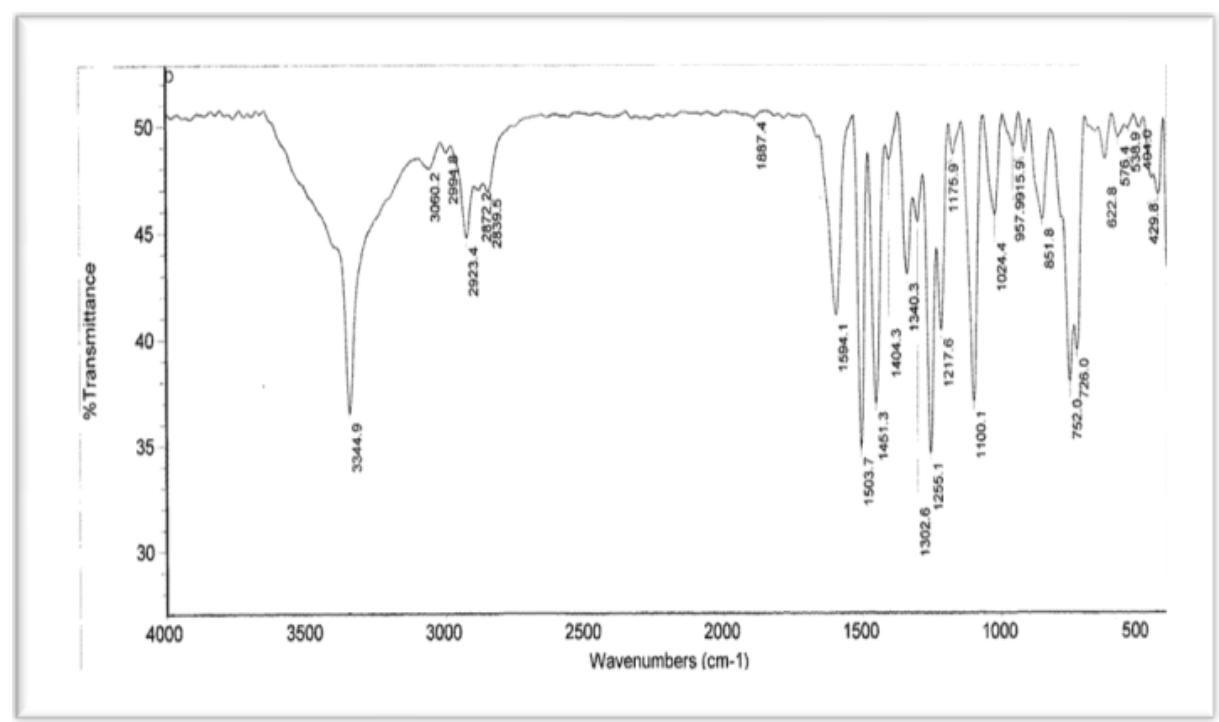

Fig. 2(B): FT-IR spectra of CAR-SA Co-crystals 


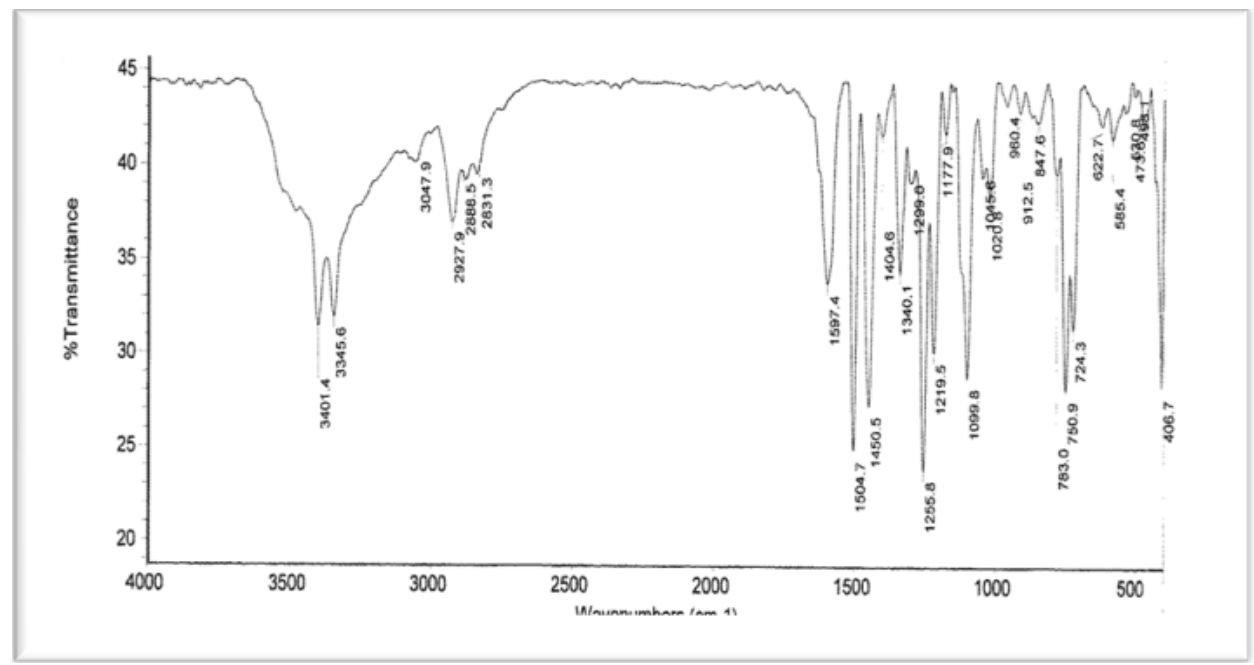

Fig. 2(C): FT-IR spectra of CAR-FA Co-crystals

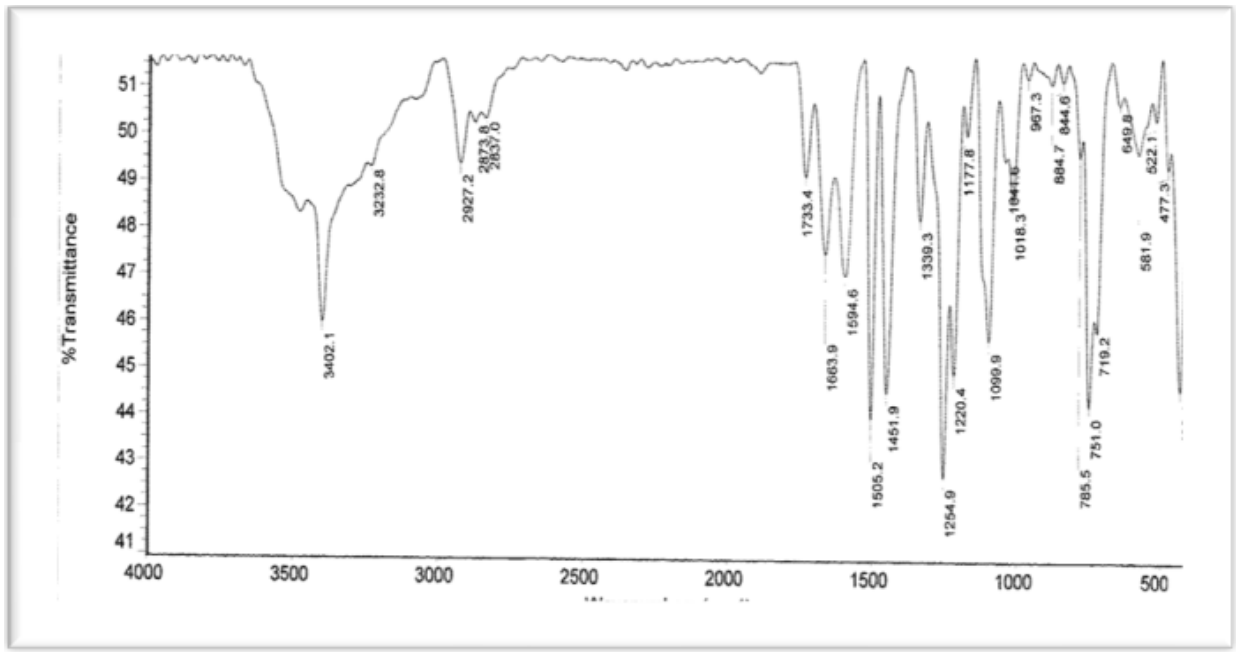

Fig. 2(D): FT-IR spectra CAR-OA of Co-crystal

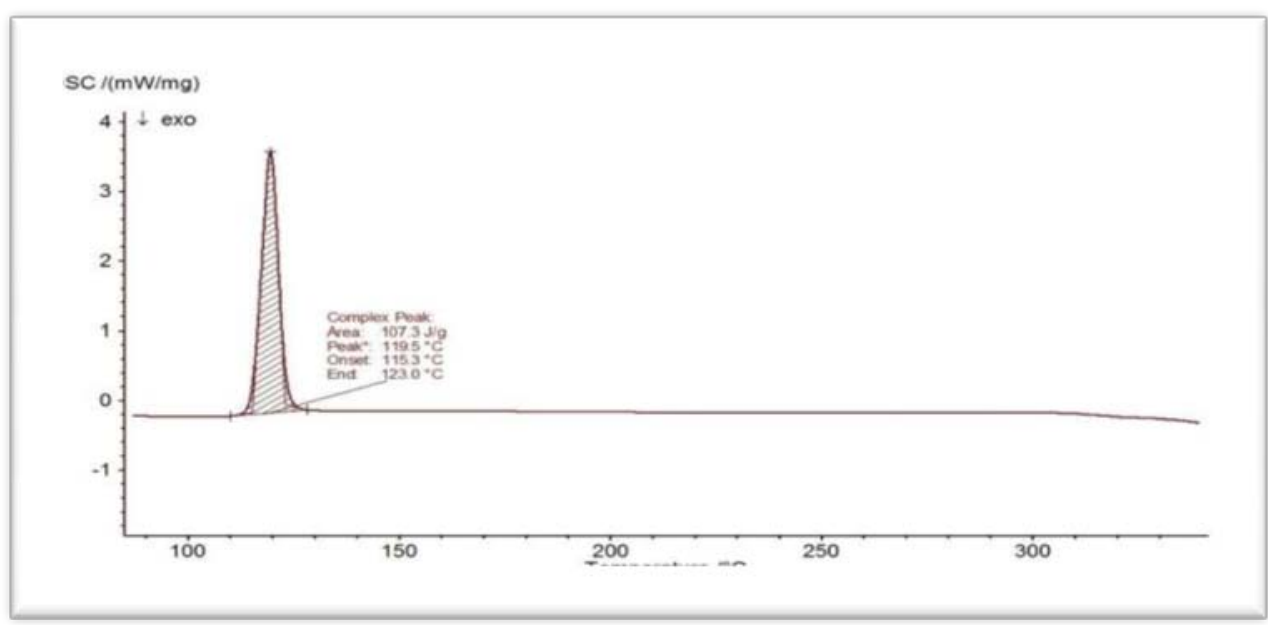

Fig. 3(A): DSC thermogram of pure carvedilol

\section{Differential scaninng calorimetry (DSC)}

DSC thermographs of pure Carvedilol and Co-crystals prepared using succinic acid, fumaric acid and oxalic acid showed a sharp endothermic peak (Tm) at $119.5{ }^{\circ} \mathrm{C}, 94{ }^{\circ} \mathrm{C}, 115{ }^{\circ} \mathrm{C}$ and $100.5{ }^{\circ} \mathrm{C}$ respectively corresponding to the melting point of Carvedilol Melting endotherm appreciably change in Carvedilol Co-crystals. This observation confirmed the formation of new solid phase and their value obtained in DSC thermogram shown in fig. 2 (A-D). The Shift of the endothermic peak towards lower temperature dictates 
decreased melting point of the drug in the Co-crystals. This decreased melting point of Co-crystals accounts for increased solubility of the drugs. The decrease in the solubility of drug in cocrystals was reported by Researcher $[17,18]$.

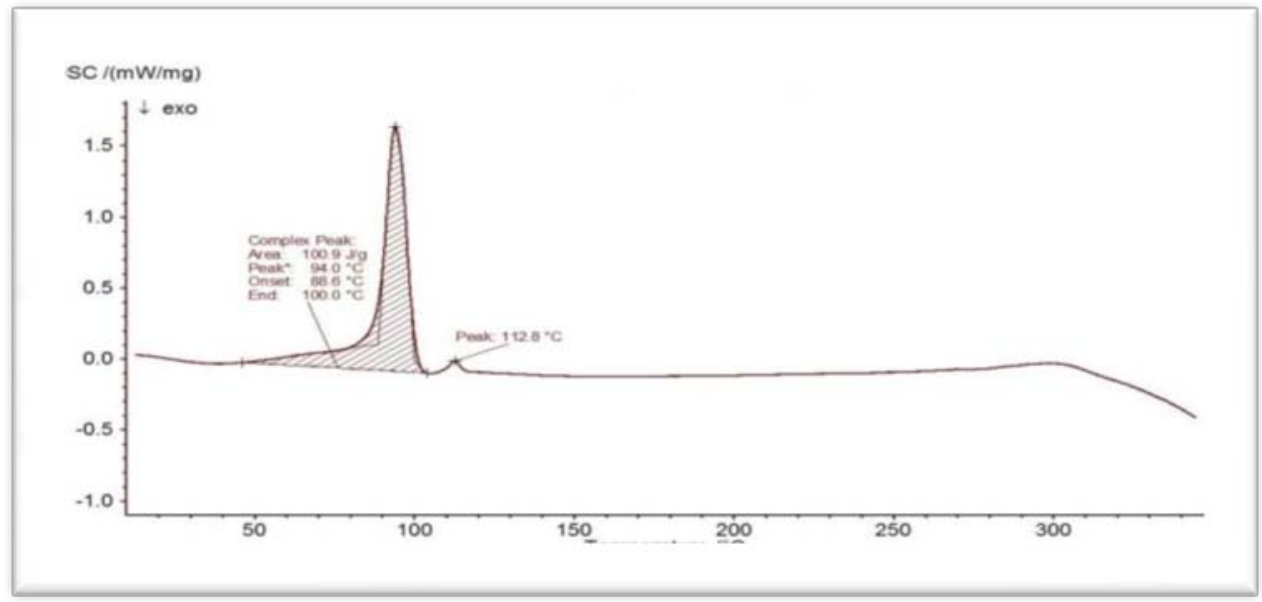

Fig. 3(B): DSC Thermogram CAR-SA Co-crystals

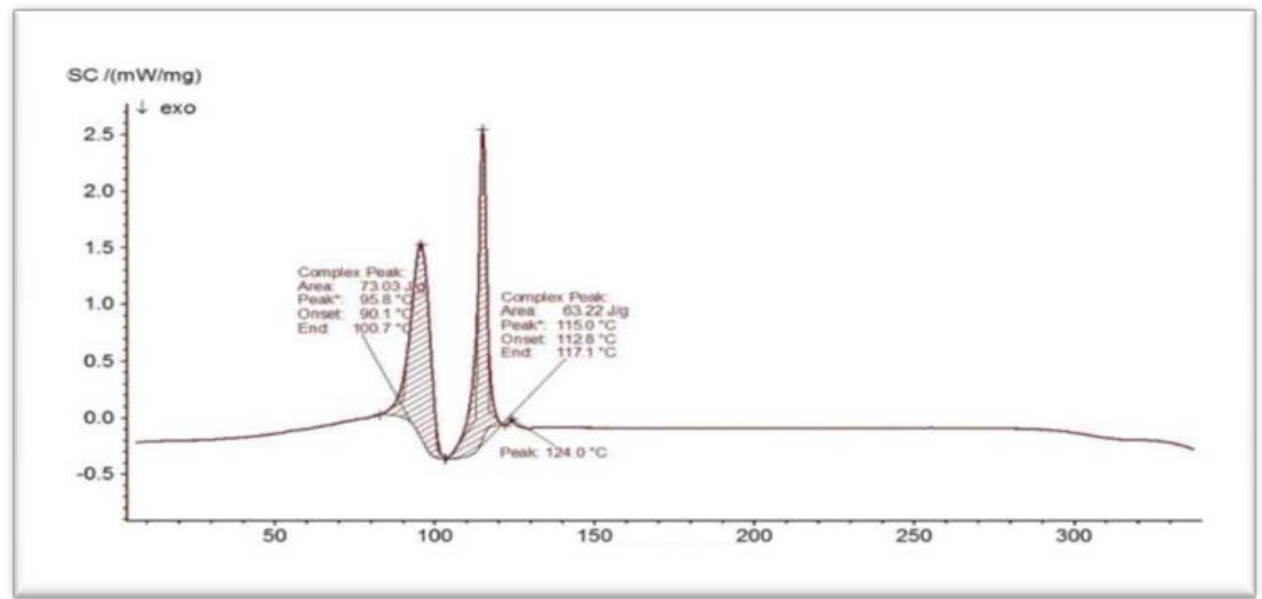

Fig. 3(C): DSC Thermogram CAR-FA Co-crystals

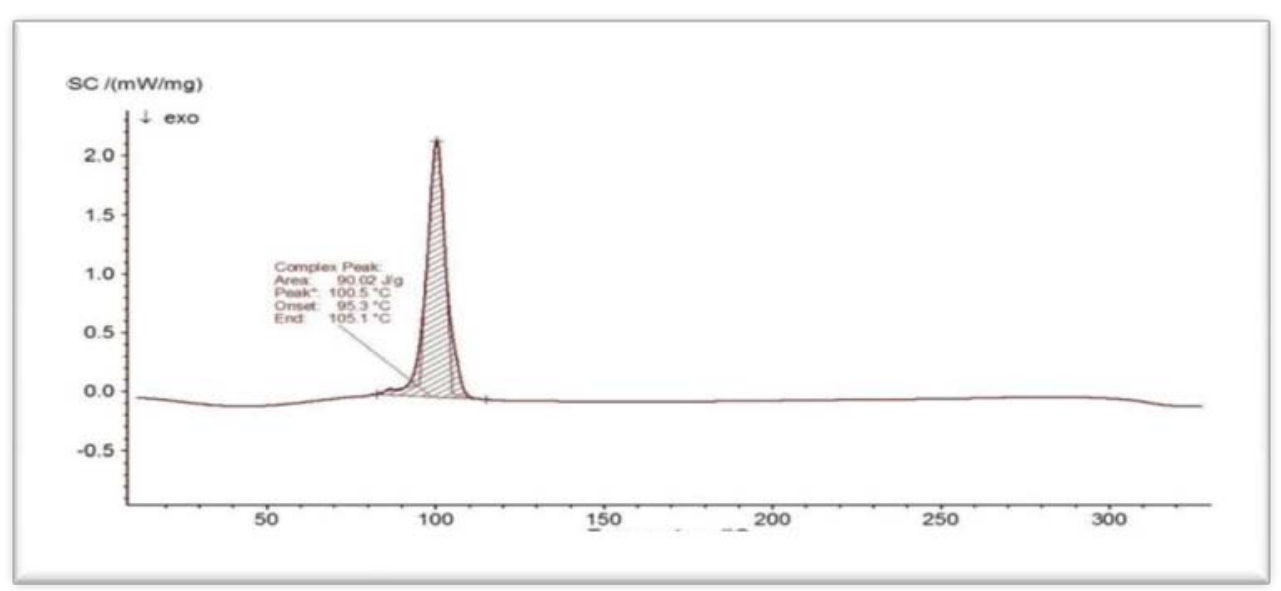

Fig. 3(D): DSC Thermogram CAR-OA Co-crystals

\section{X-ray diffraction}

XRD pattern of Pure Carvedilol fig. 4 (A) showed intense and sharp peaks indicating its crystalline nature. The diffractogram of the Cocrystals with succinic acid, fumaric acid and oxalic acid shown in fig. 4 (B)-(D) From XRD spectra it was observed and concluded that, the intensity and number of peaks were reduced in the XRD spectra of Carvedilol co- crystals compare to pure Carvedilol indicate that the reduction in crystallinity and formation of new bonding in the co-crystals This variation in the XRD pattern of pure Carvedilol and its Co-crystals due to changes in the crystal lattice structure of drug and conformer [19].

It means that Co-crystals with these conformers enhance solubility and, ultimately the dissolution by retaining crystallinity of the drug. 


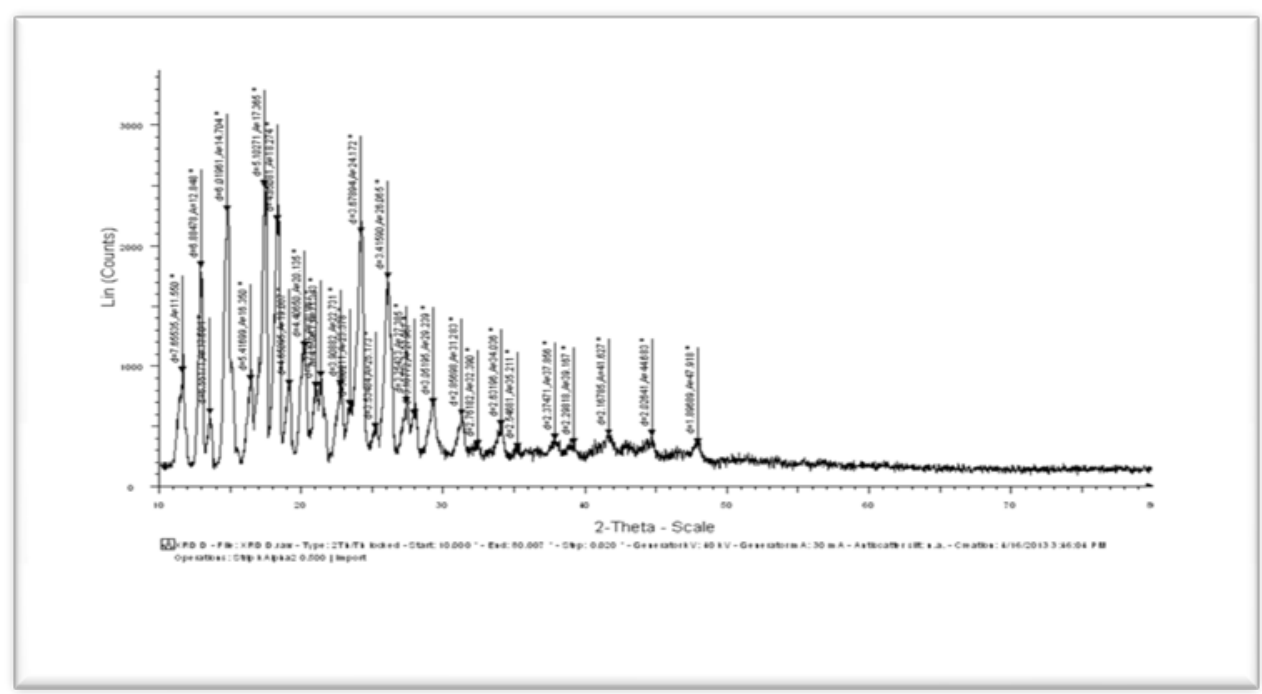

Fig. 4(A): XRD spectra of pure carvedilol

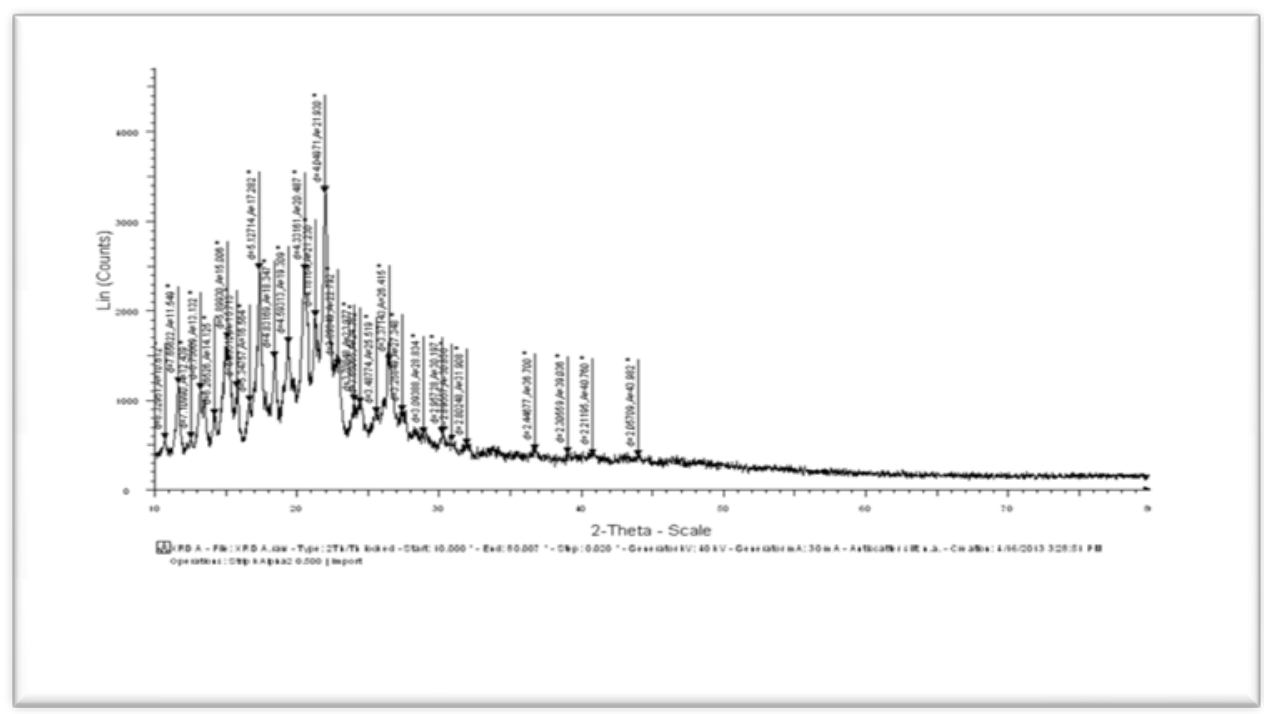

Fig. 4(B): XRD spectra CAR-SA Co-crystals

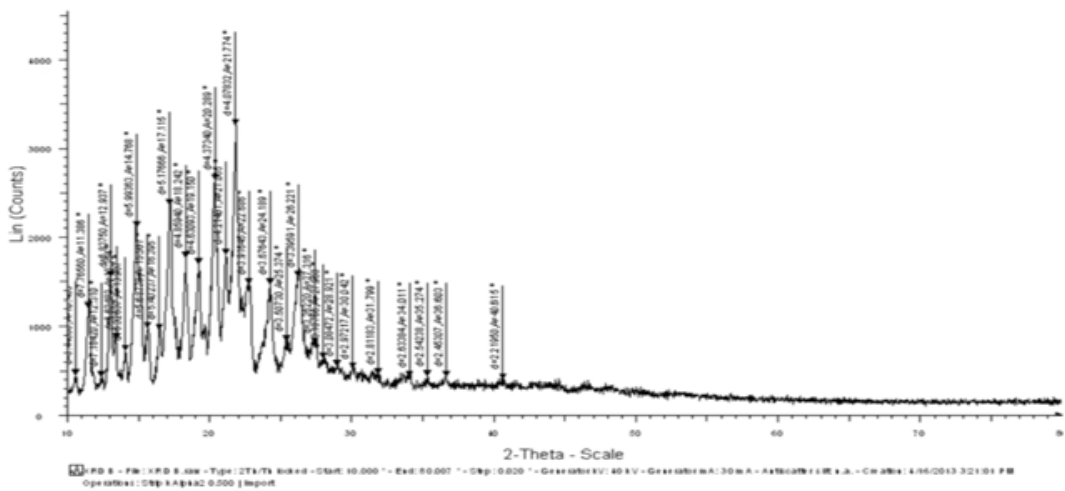

Fig. 4(C): XRD Spectra CAR-FA Cocrystals, This is the file that I received from where I send samples for characterization. This is the good quality of picture that $I$ have please consider 


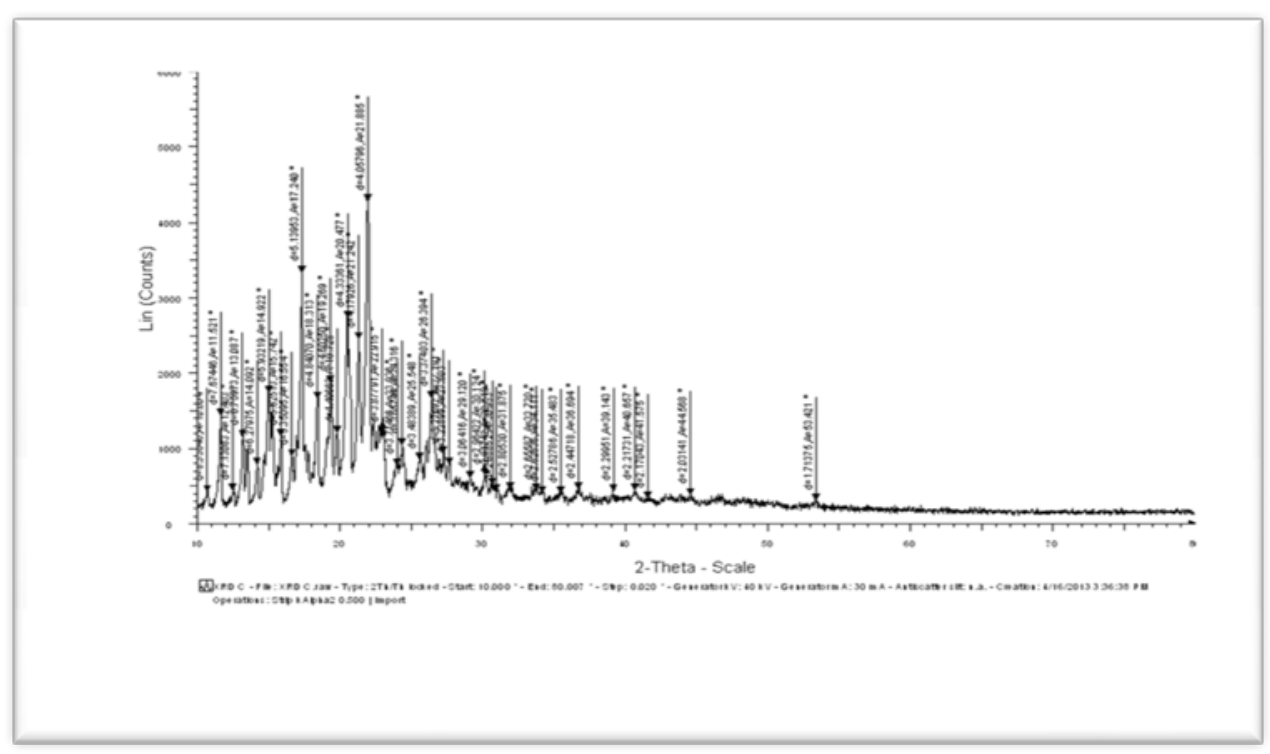

Fig. 4(D): XRD Spectra CAR-OA cocrystals

\section{Solubility study}

Pure Carvedilol shows poor aqueous solubility $(0.376(\mu \mathrm{g} / \mathrm{ml})$. CAR-SA Co-crystals showed higher solubility compare to CAR-OA and CAR-FA Co-crystals. This significant improvement in the aqueous solubility of Co-crystals of Carvedilol is due to the formation of new solid-phase between drug and conformer [20]. The order of increased solubility was CAR-SA Co-crystals>CAR-FA Co-crystals>CAR-OA Co-crystals. The CAR-SA Co-crystals increased six-fold solubility, CAR-FA Co-crystals increased fivefold solubility and CAR-OA Co-crystals increased threefold solubility compared to the pure Carvedilol. The solubility data of pure Carvedilol and its Co-crystals were shown in table 2.

Table 2: Solubility studies of pure carvedilol and its co-crystals

\begin{tabular}{llll}
\hline S. No. & Formulation code & Saturation solubility in distilled water $(\boldsymbol{\mu g} / \mathbf{m l})$ & Increase in solubility \\
\hline 1 & Pure Carvedilol & $0.376 \pm 0.06$ & -- \\
2 & CAR-SA Co-crystals & $2.225 \pm 0.35$ & Six fold \\
3 & CAR-FA Co-crystals & $1.880 \pm 0.20$ & Five fold \\
4 & CAR-OA Co-crystals & $1.128 \pm 0.23$ & Three fold \\
\hline
\end{tabular}

Standard deviation $(\mathrm{n}=3)$

\section{Micromeritic studies}

The micromeritic properties such as flowability of Co-crystals are shown in Table.3. The flowability represented in terms of the angle of repose, Carr's index and Hausner' ratio. The Co-crystals prepared using succinic acid, fumaric acid and oxalic acid showed improved micromeritic properties compared to pure Carvedilol. The Hausner ratio for cocrystals with succinic acid, fumaric acid and oxalic acid was found to be less than 1.25 whereas pure carvedilol has greater than 1.25 indicating improvement in their flow properties. The angle of repose of Carvedilol Co-crystals in the range of 24.68-25.85 compared to pure Carvedilol 38.49. The cars index for Carvedilol cocrystals were in the range of 14.33-17.45. The value of Carr's index indicates the better flowability compare to pure Carvedilol (34.49) these findings proved that the flowability of Co-crystals was preferably improved as compared to pure Carvedilol crystals.

Table 3: Micromeritic properties of pure carvedilol and cocrystals

\begin{tabular}{lllll}
\hline Formulation & Bulk density $(\mathrm{g} / \mathbf{m l})$ & Tapped density $(\mathrm{g} / \mathrm{ml})$ & Angle of repose $\left(^{\circ}\right)$ & Carr's index $(\%)$ Hausner ratio \\
\hline Pure Carvedilol & $0.56 \pm 0.05$ & $0.85 \pm 0.03$ & $38.49 \pm 1.5$ & $34.49 \pm 1.79$ \\
CAR-SA Co-crystals & $0.60 \pm 0.01$ & $0.68 \pm 0.02$ & $24.68 \pm 0.9$ & $1.52 \pm 1.2$ \\
CAR-FA Co-crystals & $0.61 \pm 0.02$ & $0.72 \pm 0.05$ & $25.85 . \pm 1.3$ & $14.33 \pm 1.35$ \\
CAR-OA Co-crystals & $0.60 \pm 0.03$ & $0.70 \pm 0.04$ & $25.10 \pm 1.2$ & $17.45 \pm 1.40$ \\
\hline
\end{tabular}

Standard deviation $(\mathrm{n}=3)$

\section{Dissolution studies}

In vitro release drug profile of Pure Carvedilol (40.30) and Co-crystals with succinic acid, fumaric acid and benzoic acid shown in fig. 5. CAR-SA Co-crystals showed $93.72 \%$ drug release, whereas CAR-FA Co-crystal and CAR-OA Co-crystals have $91.56 \%$ and $88.93 \%$ drug release in 60 min respectively. The drug release data of Carvedilol Co-crystals showed a dramatic enhancement in dissolution rate compared to pure Carvedilol. This increase in the release pattern was due to the decrease in crystallinity and formation of new solid phase. 


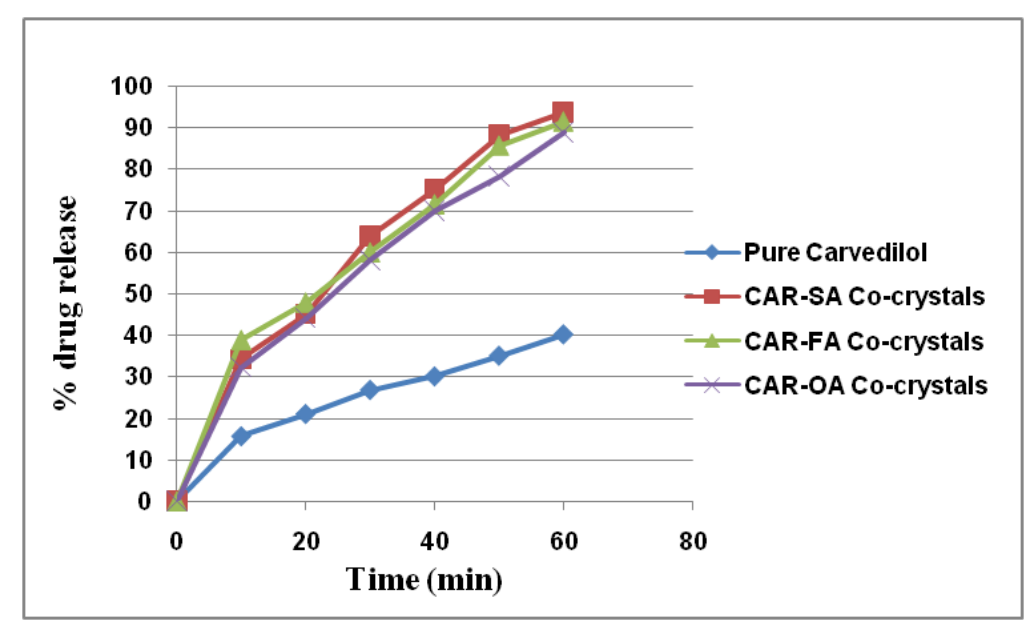

Fig. 5: Dissolution profile of pure carvedilol and Co-crystals (All the values were calculated as mean $\pm \mathrm{SD}$ ) $\mathrm{n}=3$

\section{CONCLUSION}

Carvedilol Co-crystals using different coformer viz. succinic acid, fumaric acid and oxalic acid were prepared by the solvent evaporation method. Co-crystals showed significant improvement in the solubility and dissolution rate compare to pure Carvedilol. The SEM, FT-IR, DSC and XRD data also support and confirmed the formation of new solid phase. Thus Cocrystals could be one of the successful techniques to improve the solubility and dissolution rate of Carvedilol.

\section{ACKNOWLEDGMENT}

The authors are thankful to Dr. Rajendra Gode College of Pharmacy, Malkapur for FT-IR and DSC studies. Author also thankful to Diya Labs, Mumbai for providing SEM, XRD studies.

\section{AUTHORS CONTRIBUTIONS}

All the authors have contributed equally.

\section{CONFLICT OF INTERESTS}

Author declares no conflict of Interest

\section{REFERENCES}

1. Aakeroy $\mathrm{CB}$, Forbes $\mathrm{S}$, Desper J. Using co-crystals to systematically modulate aqueous solubility and melting behavior of an anticancer drug. J Am Chem Soc 2009;131:17048-9.

2. Ramanathan T. Ed. Mass spectrometry in drug metabolism and pharmacokinetics. John Wiley and Sons, Inc., Hoboken, NJ; 2009. p. 1-4.

3. Rasenack N, Hartenhauer H, Muller BW. Microcrystals for dissolution rate enhancement of poorly water-soluble drugs. Int J Pharm 2003;254:137-45.

4. Hong JY, Kim JK, Song YK, Park JS, Kim CK. A new selfemulsifying formulation of itraconazole with improved dissolution and oral absorption. J Controlled Release 2006; 10:332-8.

5. Chowdary KPR, Pavan Kumar A. Recent research on formulation development of BCS class II drugs, a review. Int Res J Pharm Appl Sci 2013;3:173-81.

6. RR Thenge, VB Patond, NM Mahajan, VS Adhao, PV Ajmire, LN Barde, et al. 'Preparation and characterization of co-crystals of diacerein. Indo J Pharm 2017;28:34-41.
7. S Mirza, I Miroshnyk, J Heinamaki, J Yliruusi. Co-crystals: an emerging approach for enhancing the properties of pharmaceutical solids. Dosis 2008;24:90-6.

8. Sevukarajan M, Tanuja B. Synthesis and characterization of pharmaceutical cocrystals. (Aceclofenac-Nicotinamide) J Pharm Sci Res 2011;3:1288-93.

9. V Yadav, AS Shete, AP Dabke, PV Kulkarni, SS Sakhare. Cocrystals: a novel approach to modify physicochemical properties of active ingredients. Ind J Pharma Sci 2009;71:359-70.

10. Rajurkar VG, Nagare AS, Ghawate V. Tablet formulation and enhancement of aqueous solubility of efavirenz by solvent evaporation co-crystal techniques. Med Chem 2015;S2:002.3

11. Instrumental methods of analysis. 7th edition. by Willard, Merritt, Dean and Settle; 1989. p. 796.

12. Palermo PJ. Handbook of modern pharmaceutical analysis. Ahuja S, Scypinski S. eds. Academic Press: San Deigo, USA; 2001. p. 243-4.

13. Yuvraja K, Khanam J. Enanhancement of carvedilol solubility by solid dispersion techniques using cyclodextrin, a water-soluble polymer and hydroxyl acid. J Pharm Biomed Anal 2014;96:510-20.

14. Thenge RR. Crystal modification of aceclofenac by spherical crystallization to improve solubility dissolution rate and micromeritic properties. J Pharma Res 2012;5:974-7.

15. Planinsek O, Kovacic B, Vrecer F. Carvedilol dissolution improvement by preparation of solid dispersions with porous silica. Int J Pharm 2011;406:41-8.

16. Hiendrawan S, Hartanti AW, Veriansyah B, Widjojokusumo E, Tjandrawinata RR. Solubility enhancement of ketoconazole via salt and cocrystal formation. Int J Pharm Pharm Sci 2015;7:160-4.

17. Chadha R, Saini A, Arora P, Chanda S, Dharamvir Singh Jain. Cocrystal of efavirenz with selected conformers: preparation and characterization. Int J Pharm Pharm Sci 2012;4:244-50.

18. Hiendrawan S, Veriansyah B, Widjojokusumo E, Soewandhi SN, Wikarsa S, Tjandrawinata RR. Simultaneous cocrystallization and micronization of paracetamol-dipicolinic acid cocrystal by supercritical antisolvent (SAS). Int J Pharm Pharm Sci 2016;8:89-98.

19. F Rahman, AN Winantari, Siswandono, D Setyawan. Comparison study of grinding and slurry method on the physicochemical characteristic of acyclovir-succinic acid cocrystal. Asian J Pharm Clin Res 2017;10:153-8.

20. M Raghuram, MS Alam, M Prasad, CH Khanduri. Pharmaceutical cocrystal of prulifloxacin with nicotinamide. Int J Pharm Pharm Sci 2014;6:180-4. 